\title{
Modelling of agricultural combination driver behaviour from the aspect of safety of movement
}

\author{
Jan Szczepaniak', Wojciech Tanaś2 ${ }^{2}$ Tadeusz Pawłowski', Jacek Kromulski ${ }^{1}$ \\ ${ }^{1}$ Industrial Institute of Agricultural Engineering, Poznań, Poland \\ 2 University of Life Sciences in Lublin, Lublin, Poland
}

Szczepaniak J, Tanaś W, Pawłowski T, Kromulski J. Modelling of agricultural combination driver behaviour from the aspect of safety of movement. Ann Agric Environ Med. 2014; 21(2): 403-406. doi: 10.5604/1232-1966.1108613

\begin{abstract}
Statistics show that the travel of agricultural machinery to a work area and their movement during labour is the source of many serious accidents. The most dangerous in consequences prove to be those that occur during transport and associated with maneuvering tractors and machinery (about $30 \%$ of all fatal accidents). It can be assumed that at least some of these accidents were caused indirectly by the specific design features of agricultural machines which adversely affect the driveability. The single- and multi-loop structures of the driver-vehicle system models are formulated to study the contributions of various preview and prediction strategies to the path tracking and dynamic performance of the articulated vehicle. In the presented study the compensatory model of driver utilizes the lateral acceleration of the tractor, roll angle of trailer sprung mass and the articulation rate as the internal motion feedback variables. The control model of steering of an agricultural set has been implemented in the Matlab/Simulink environment. The model has been constructed with the use of stochastic methods and operational transmittances describing the various components of the system. The model operational transmittances has been estimated using Box-Jenkins and continuous-time process models from input-output data. The model has been tested using experimental data from road investigation of the agricultural set.
\end{abstract}

Key words

human operator, vehicle-driver model, simulation

\section{INTRODUCTION}

From among the three components of the driver-vehicleenvironment system, the driver is the most unsafe part of this system. In this case, a better understanding of the drivervehicle control system, which makes it possible to adapt the agricultural combination construction to psychophysical characteristics of the driver, is a method for improving the active safety of the agricultural combination.

Statistics show that the travel of agricultural machinery to the work area and their movement during labour is the source of many serious accidents. The most dangerous in consequences prove to be accidents occurring during transport and associated with maneuvering tractors and machinery (about 30\% of all fatal accidents). In 2007 - 2011, 1,956 accidents were reported in Poland to the Agricultural Social Insurance Fund (KRUS) (including 171 fatal accidents) in the group: drive-over, crash, seizure by means of transport on the move.

It can be assumed that at least some of these accidents were caused indirectly by the specific design features of agricultural machines which adversely affect the driveability. Analysis of steerability and stability of agricultural machinery needs to take into account the specific features of the construction of agricultural machines, their connection to the tractor and the behaviour of the machine under different operating conditions (field, road) with complex and variable characteristics. Taking into account the specific features of

Address for correspondence: Wojciech Tanaś, University of Life Sciences, Akademicka 13, 20-950 Lublin, Poland

e-mail:wojciech.tanas@up.lublin.pl

Received: 02 July 2012; accepted: 17 April 2013 agricultural machinery in the simulation analysis of vehicle steerability and stability, these should be implemented already in the early stages of design engineering

Control model of the human operator. Vehicle dynamics is one of the most important fields in research of motorization engineering. Running, acceleration, braking, changing of motion direction are typical dynamic processes. Their quality of their execution depends on the driver. A vehicle operated by driver may be considered as a control system with driver as controller and vehicle as the controlled system. Driver efficiency depends on the situation on the road, vehicle characteristics as well as psychical and physical driver features. From a linear input-output system viewpoint, the vehicle may be considered as a generalized plant with the driver acting as the feedback control element. The driver regulates the system outputs (lateral velocity and yaw rate) in order to follow a desired roadway path. The main objective of the control of vehicle dynamics is to improve the handling performance, or maneuverability, in order to obtain safer and happier driving.

The mathematical model of a vehicle takes into consideration the driver activity which consists of two main parts: driver model and vehicle model $[1,2]$. The connections between road, driver and driven vehicle are shown in Figure 1. A set of mathematical equations in the analytical model is used to predict the important operating parameters of the vehicle's steerability [3]. Identification of the driver model parameters is achieved through an optimal control approach. [4]. Parameters describing drives behaviour could be identified based on a system model in the frequency domain (from power spectral density) or in the time domain (from correlation). 


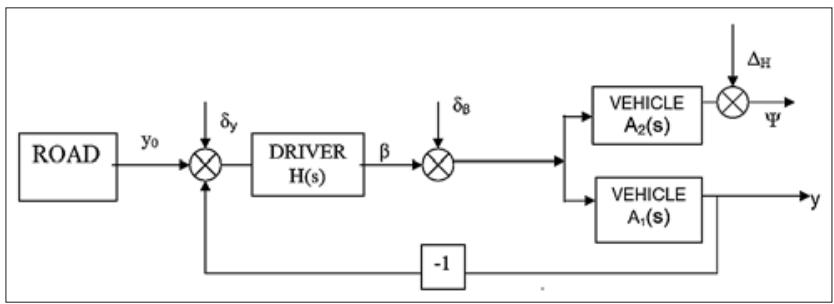

Figure 1. Simplified diagram of road-driver-vehicle system with feedback

Steering angle $\beta(\mathrm{t})$, vehicle yaw angle $\Psi(\mathrm{t})$ and its lateral displacement $y$ are specified by the equation (with initial condition $y=0)[5]$.

$$
\left\{\begin{array}{l}
\beta=H^{*} \delta_{y}-H^{*} y \\
y=A_{1} * \delta_{\beta}+A_{1}^{*} \beta \\
\Psi=A_{2} * A_{1}^{-1} * y+\Delta_{H}
\end{array}\right.
$$

It is possible to obtain dependences characterized by transmittance $\mathrm{H}$ of the driver model on the basis of above equation, spectral density, and reciprocal spectral density of the measured signals:

$$
\delta_{\mathrm{y}}{ }^{*} \delta_{\mathrm{y}}{ }^{*}, \delta_{\beta}{ }^{*} \delta_{\beta}{ }^{*} \Delta_{\mathrm{H}}{ }^{*} \Delta_{\mathrm{H}}{ }^{*}, \delta_{\beta}{ }^{*} \delta_{\mathrm{y}}{ }^{*}, \Delta_{\mathrm{H}}{ }^{*} \delta_{\mathrm{y}}^{*} .
$$

The transmittance $A_{1}$ and $A_{2}$ are dependent only on the physical parameters of the vehicle. It is possible to estimate the transmissibility function or autocorrelation function of the driver depending on the driver's response time delay $(\tau)$, and constant parameters depending on the driver's reaction time [6].

A comprehensive closed-loop driver-articulated vehicle model. The directional performance behaviour of the coupled articulated vehicle and driver system is dependent upon the path preview, and driver's perception of directional response of the leading and trailing units.

Figure 2 presented the multi-loop structure of the compensatory model of employing the driver's perception of the articulation rate, roll angle of the trailer sprung mass and lateral acceleration of the tractor [7].

The preview block $\mathrm{P}(\mathrm{s})$ represents the preview function (preview strategy), which is assumed to be an effective single point preview strategy, in that:

$$
P(s)=e^{T_{p} s}\left(1-\frac{\lambda T_{p} s}{3}\right)
$$

The low frequency compensatory function $\mathrm{Hc}(\mathrm{s})$, has the form:

$$
H c(s)=K_{C}\left(1+T_{C} s\right) e^{-\tau_{d} s}
$$

where $K_{c}$ is the driver's compensatory gain, $T_{c}$ is the driver's correction time constant and $\tau_{\mathrm{d}}$ is the driver's reaction time.

The components of the compensatory function of the driver $\mathrm{HH}$, can be defined as:

$$
H H(s)=K_{H} e^{-\tau_{H} s}
$$

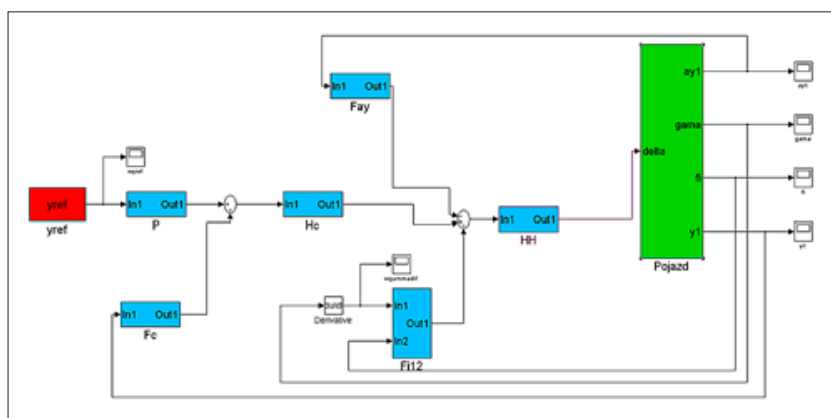

Figure 2. A Matlab/Simulink model of the multi-loop structure of the compensatory driver model

The function $\mathrm{HH}$ represent the driver's neuromuscular dynamics, and the proprioceptive feedback elements derived from the motion of the human limbs and muscle tissues (Yang, 1999). The function Fc, representing the driver's path prediction, is given by:

$$
F c(s)=1+T_{P} s+\frac{T_{P}^{2} s^{2}}{2}+\frac{\lambda T_{P}^{3} s^{3}}{6}
$$

The function Fi12 and Fay represent the motion feedback from the roll angle of the sprung mass of the trailer, articulation rate between the two units. and the lateral acceleration:

$$
\begin{gathered}
F \gamma(s)=K_{\gamma} e^{\tau_{\gamma} s} \\
F \phi(s)=K_{\phi} e^{\tau_{\phi} s} \\
F a y(s)=K_{a y 1} e^{\tau_{a y 1} s}
\end{gathered}
$$

$K_{\phi}$ and $\tau_{\phi}$ are the driver's gain and time delay corresponding to prediction of the roll angle response of the trailer sprung mass, while $K_{\gamma}$ and $\tau_{\gamma}$ are the driver's gain and time delay corresponding to prediction of the articulation rate response of the trailer combination; $K_{a y}$ and $\gamma_{a y}$ are the driver's prediction gain and delay time for the lateral acceleration of the trailer sprung mass.

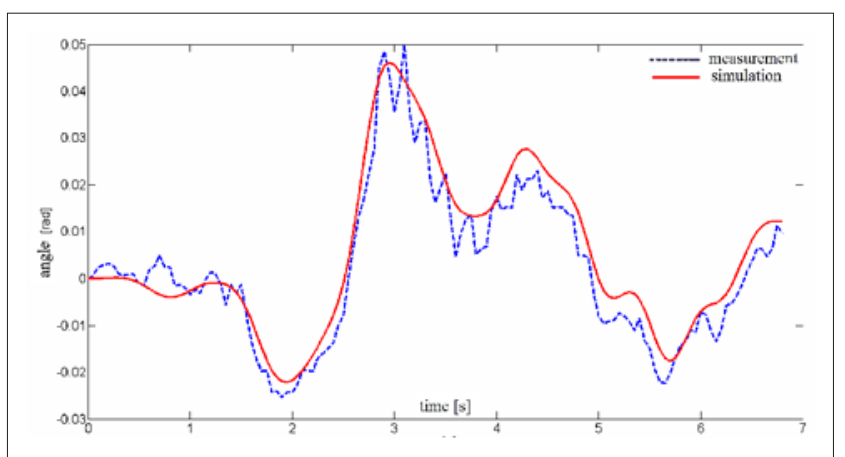

Figure 3. Comparison of the angle between trailer and vehicle designated experimentally and by simulation

Identification of driver delay reaction time. In psychological studies, the driver reaction process is further represented in four states: perception, recognition, decision and physical response. Many studies have estimated the reaction time based on indoor experiments and driving simulators. To estimate driver reaction delays from real data, several approaches have been proposed. For example, in the study by Johansson and 
Rummerm more than 300 subjects were instructed to use the brake pedal as soon as they heard a certain sound. Pawlowski [6] developed a method to identify the individual driver reaction time based on autocorrelation function.

The equations of the system shown in Figure 1 have the form [5]:

$$
\begin{gathered}
\beta=G_{2}\left(A_{1}^{-1} \delta_{y}-\delta_{\beta}\right) \\
y=G_{2}\left(H^{-1} \delta_{\beta}-\delta_{y}\right) \\
\psi=p y / v_{a}
\end{gathered}
$$

where $\mathrm{p}=\mathrm{d} / \mathrm{d} t$.

Obtained expressions for power spectral densities:

$$
\begin{gathered}
G_{\beta \beta}=G_{\delta_{\beta}}\left|G_{2}\right|^{2}\left[G_{\delta_{y}} G_{\delta_{\beta}}^{-1}\left|A_{1}\right|^{-2}+1\right] \\
G_{\psi \psi}=G_{\delta_{\beta}} \omega^{2} v_{a}^{-2}\left|G_{2}\right|^{2}\left[|H|^{-2}+G_{\delta_{y}} G_{\delta_{\beta}}^{-1}\right] \\
G_{\psi \beta}=G_{\delta_{\beta}} j \omega v_{a}^{-1}\left|G_{2}\right|^{2}\left[H^{*-1}-G_{\delta_{y}} G_{\delta_{\beta}}^{-1} A_{1}^{-1}\right]
\end{gathered}
$$

The Power Spectral Density for steering angle $\beta(t)$ can also be expressed as an equation:

$$
G_{\beta \beta}=G_{\delta_{\beta}}\left(1+b^{2} T_{\beta}^{2} \omega^{2}\right) /\left(1+T_{\beta}^{2} \omega^{2}\right)^{3}
$$

where

$$
b=T_{\beta}^{-1}\left(\tau+3 T_{\beta}+\sqrt{2} T_{\delta}\right)
$$

The Wiener-Khintchine theorem states that the autocorrelation function is given by the Inverse Fourier Transform of the Power Spectral Density (PSD). The autocorrelation function can be defined by [5]:

$$
k_{\beta \beta}(\theta)=\frac{1}{2 \pi} \int_{-\infty}^{+\infty} G_{\beta \beta}(\omega) e^{i \omega \theta} d \omega
$$

An expression for the normalized autocorrelation function for steering angle $\beta(t)$ is derived below:

$$
\rho_{\beta \beta}(\theta)=e^{-\left|\frac{\theta}{T_{\beta}}\right|}\left[1+\left|\frac{\theta}{T_{\beta}}\right|+\frac{1-b^{2}}{3+b^{2}}\left(\left|\frac{\theta}{T_{\beta}}\right|\right)^{2}\right]
$$

The optimisation method can be used for identifying real driver parameters. Driver's reaction time can be determined also from the transmittance $\mathrm{HH}$ (eq.4).

Identification of driver model from experimental data. Experimental research was conducted to obtain information about the behaviour of the unit while driving at different speeds, and performing various maneuvers. These studies were performed for different drivers $[8,9]$. Parameters of the maneuvers performed (speed, lane width, radius turns, etc.) were consistent with the recommendations of the ISO standards.

The transmittances of driver $\mathrm{H}$ for different driver and vehicle way were investigated. The optimization method was carried out for identification of real driver parameters (time parameters and reaction time delay $\tau$ ) using the Nelder-Mead simplex algorithm [10]. Analysis of the control system was carried out using MATLAB analysis software [11].

Figure 5 shows the plots of normalized autocorrelation functions of steering angle $\beta$ during testing for four different drivers.

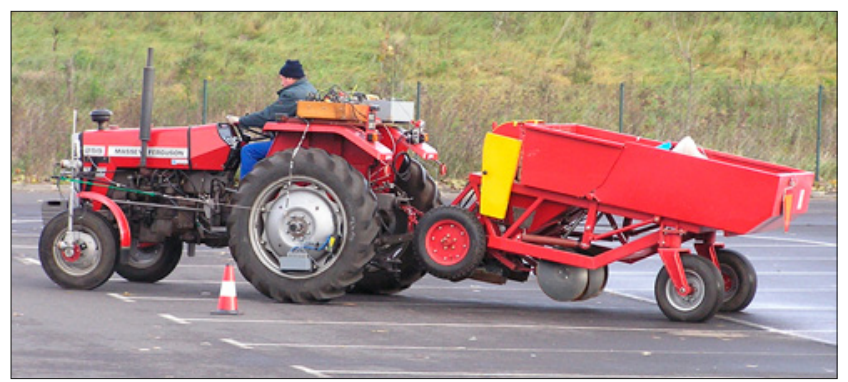

Figure 4. Semi-mounted potato planter during field work

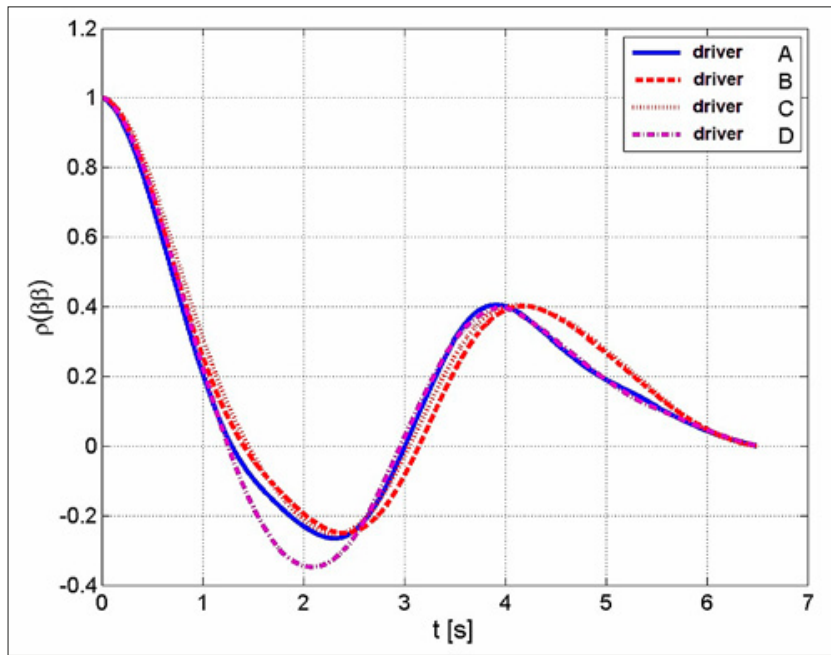

Figure 5. Correlogram comparison for different drivers: $A, B, C$ and $D$

Calculated for individual driver's reaction time $\tau$ using the Nelder-Mead simplex algorithm was different. The driver reaction time for individual drivers ranged from 0.31 (driver C) -0.38 (driver A and D) seconds.

\section{CONCLUSIONS}

The study presents initial conception taking into consideration a driver working in a tractor - agricultural machine set model. The vehicle operated by a driver may be considered as a control system with the driver as controller and the vehicle as the controlled system. Parameters describing drivers' reaction time could be identified based on the control system model in the frequency domain (from transmissibility), or in the time domain (from correlation). Conducted tests proved that the proposed model described well the tractorpotato planter steerability. The developed driver model can be used in studies of the stability and steerability of agricultural machines. The model can also be used for studies of the influence of different parameters on the stability and steerability of the driver-vehicle system, and in this way for a better adaptation of vehicle dynamic properties to driver characteristics.

\section{Acknowledgement}

This work was supported by National Science Centre Research Project No. N N313 789040 


\section{REFERENCES}

1. Doman D. Projection Methods for Order Reduction of Optimal Human Operator Models, PhD Thesis, Faculty of the Virginia Polytechnic Institute and State University, Blacksburg, Virginia 1998.

2. Reński A. Modelling of driver behaviour in a driver - vehicle environment system. Prace Naukowe, Mechanika. Politechnika Warszawska, Warszawa 2000.

3. Schafer FR. Generalized Feedback Control and Application to Vehicle Path Following Control. TU, Berlin 2004

4. Szczepaniak C. Basics of modelling the system man - vehicleenvironment. PWN, Warszawa1999.

5. Chaczaturow AA. Dynamics of the road-tire-car-driver system. Maszinostrojenie. Moskwa 1976.

6. Pawłowski T, Kromulski J, Szczepaniak J. Influence of driver perception on safety of movement of agricultural machines Transport Przemysłowy 2008; 1(31): 76-78
7. Yang X. A closed-loop driver/vehicle directional dynamics predictor PhD Thesis, Department of Mechanical Engineering, Concordia University Montreal, Quebec, Canada1999.

8. Tajanowskij G, Tanas W. The analysis of regular wheel loadings distribution at a statically unstable running system if an agricultural machine on rough surface. Teka commission of motorization and power industry in agriculture, Polish Academy of sciences branch in Lublin 2010; 10: 464-474.

9. Tajanowskij G, Tanas W. Dynamic potential of passableness of the agricultural traction-transport technological machine with a hydrodrive of wheels. Teka commission of motorization and power industry in agriculture, Polish Academy of sciences branch in Lublin 2011; 11c: 306-319.

10. Kromulski J, Pawłowski T, The modeling and control of the agricultural set, 7th International Workshop on Modeling \& Applied Simulation MAS 2008; Sept 17-19 2008; Campora S. Giovanni (Amantea, CS), 2008.

11. Ljung L, Glad T. Modeling Of Dynamic Systems. Prentice Hall, 1994. 